\title{
Misdirecting methylation to drive oncogenesis
}

Research using mice and human tumour samples has identified a potential mechanism that drives chondroblastoma and sarcoma development. Lysine-to-methionine mutations in histone $\mathrm{H} 3$ at position 36 (H3K36M) inhibit the activity of methyltransferases, leading to global changes in methylation patterns. These changes, in turn, increase the expression of genes that might block differentiation of mesenchymal progenitor cells (MPCs).

Although histone $\mathrm{H} 3$ mutations have been identified in various human cancers, whether these mutations promote the development of bone and cartilage malignancies remains unclear. H3K36M was previously identified by whole-genome sequencing as the only recurrent mutation present in human chondroblastomas, which result from impaired differentiation of MPCs into chondrocytes.

Lu et al. investigated the effect of this mutation on differentiation using isogenic $\mathrm{H} 3 \mathrm{~K} 36$ wild-type and H3K36M mutant mouse MPCs.

these

methylation

changes

were driven

by impaired

methyl-

transferase

function
Compared with wild-type MPCs, H3K36M MPCs showed defects in chondrocyte, adipocyte and osteocyte development, aberrant expression of genes involved in differentiation along these lineages and increased expression of transcription factors that promote mesenchymal multipotency. In addition, when injected into mice, H3K36M MPCs formed tumours that closely resembled human undifferentiated sarcomas, and screening of 10 human undifferentiated sarcomas identified samples carrying amino acid substitution mutations at $\mathrm{H} 3 \mathrm{~K} 36$.

The block in differentiation induced by $\mathrm{H} 3 \mathrm{~K} 36 \mathrm{M}$ correlated with alterations in histone methylation. Chromatin immunoprecipitation followed by DNA sequencing (ChIP-seq) of MPCs showed that H3K36M was associated with decreased dimethylation and trimethylation at $\mathrm{H} 3 \mathrm{~K} 36$ (H3K36me2/3) and increased trimethylation at H3K27 (H3K27me3), with $\mathrm{H} 3 \mathrm{~K} 27 \mathrm{me} 3$ hypermethylation confined to intergenic regions. This methylation pattern was similar to that found in human chondroblastoma samples carrying H3K36M.

The authors hypothesized that these methylation changes were driven by impaired methyltransferase function. Indeed, in vitro activity assays showed that the H3K36 methyltransferases nuclear receptor binding SET domain protein 1 (NSD1), NSD2 and SET domain containing 2 (SETD2) were inhibited by various mutations at $\mathrm{H} 3 \mathrm{~K} 36$, and depletion of all three methyltransferases impaired MPC differentiation and produced a histone methylation pattern similar to that induced by H3K36M.

Polycomb repressive complex 1 (PRC1) might provide a mechanistic link between these global histone methylation changes and tumorigenesis. PRC1 binds to H3K27me3 to suppress the expression of genes that have been implicated in regulating the

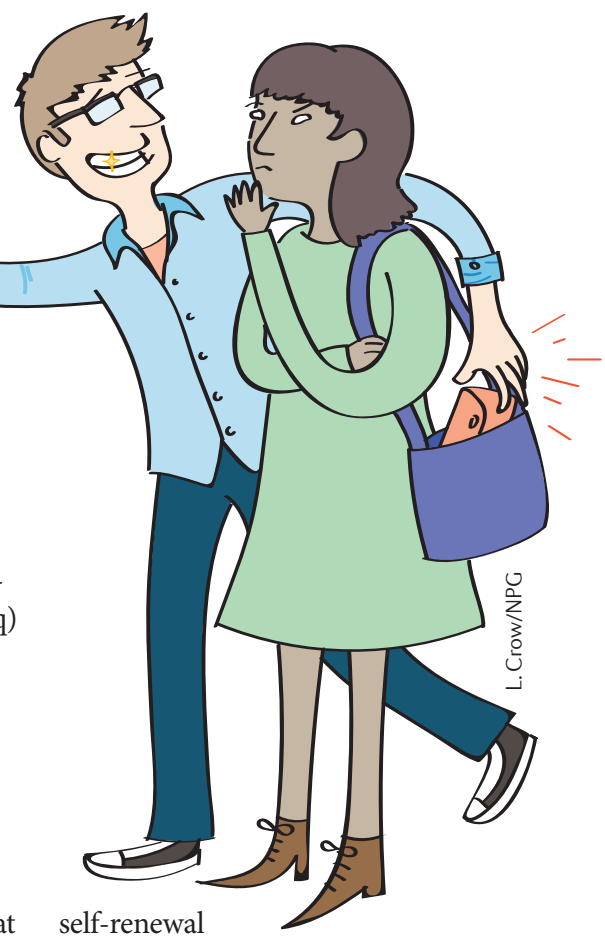

capacity of mesenchymal stem cells. In H3K36M MPCs, binding of PRC1 components to newly generated intergenic H3K27me3 sites was enriched, whereas binding to normal target genes was reduced - in effect 'diluting' the activity of PRC1 across the genome and potentially producing a block in differentiation. In the future, validation of this relationship between polycomb complex recruitment and progenitor cell differentiation more generally might indicate that a single histone mutation can initiate a cascade of changes that essentially 'locks' chromatin into an oncogenic state.

\section{Elizabeth Zuccala,} Associate Editor, Nature Reviews Disease Primers

ORIGINAL ARTICLE Lu, C. et al. Histone H3K36 mutations promote sarcomagenesis through altered histone methylation landscape. Science 352, 844-849 (2016) 